\title{
ALMOST PERIODIC FUNCTIONS
}

\author{
J. E. L. PECK
}

One of the aims in the study of almost periodic functions on a group is to generalize the Fejér summation process, whereby the Fourier series may be summed to yield the function. This has been achieved partially by Bochner-von Neumann [1] and Maak $[2 ; 3] .^{1}$ But in [3] Maak says "In general it is not possible to give a summation procedure which may be successfully applied to all almost periodic functions on the group."

This paper is intended to show that we may indeed find a generalized Fejér kernel and a summation process, which may be applied equally to all almost periodic functions on a group. We prove this for the case of complex-valued almost periodic functions on an arbitrary group. The procedure also works for functions with values in a linear topological space, but we shall not concern ourselves here with the more general case. In order to proceed to the heart of the matter, we shall assume the definitions and results of [1] and [4].

We shall denote an irreducible, normal, unitary representation [4, Definitions 9 and 10] of a group $G$ by $D$. The image of $x \in G$ is thus a square matrix $D(x)=\left(D_{\rho, \sigma}(x)\right)$ where $\rho, \sigma=1, \cdots, s^{D}$. Each $D_{\rho, \sigma}$ is an almost periodic function on $G$ [4, Theorem 19]. Let $f$ be a complex-valued almost periodic function on $G$. The Fourier coefficients of $f$ are defined by

$$
C_{D, \rho, \sigma}(f)=M_{x}\left\{D_{\sigma, \rho}\left(x^{-1}\right) f(x)\right\}
$$

$[1$, Definition 8$]$, and the formal sum

$$
\sum_{D} s^{D} \sum_{\rho, \sigma}^{\dot{s}_{1}^{D}} C_{D, \rho, \sigma}(f) D_{\rho, \sigma}
$$

is called the Fourier expansion of $f$. If $f$ and $g$ are almost periodic functions, we write $f \times g(x)=M_{\nu}\left\{f\left(x y^{-1}\right) g(y)\right\}$ and $f \times g$ is also an almost periodic function [1, Definition 6].

Our task is to give a method whereby (2) may be summed to yield the function $f$ in the sense of the topology in the space of bounded complex-valued functions on $G$ introduced by the metric

$$
|f-g|=\sup _{x \in G}|f(x)-g(x)| .
$$

Received by the editors March 8, 1951 .

${ }^{1}$ Numbers in brackets refer to the references cited at the end of the paper. 
TheOREM. Let $\mathcal{D}$ be any set of irreducible, normal, unitary, and mutually nonequivalent representations of a group $G$. Let $f$ be an almost periodic function on $G$ in whose Fourier expansion appear only representation elements from the set $\mathcal{D}$. Then we may find a directed set [5] $\left\{\phi_{\gamma} \mid \gamma \in \Gamma\right\}$ of special weight functions (that is, non-negative almost periodic functions, with mean value 1, having a finite Fourier expansion $\left[1\right.$, Definition 7]) such that $\lim _{\gamma \in \Gamma} \phi_{\gamma} \times f=f$, where this is a directed limit over the directed index set $\Gamma$. More specifically, there is a set of real numbers $\left\{r_{D}^{\gamma}, \gamma \in \Gamma, D \in \Phi\right\}$ such that $0 \leqq r_{D}^{\gamma} \leqq 1, \lim _{\gamma \in \Gamma} r_{D}^{\gamma}=1$, for each $\gamma$ only finitely many are nonzero, and

$$
\phi_{\gamma} \times f=\sum_{D \in \mathcal{D}^{r}} r^{\gamma} s^{D} \sum_{\rho, \sigma}^{s^{D}} C_{D, \rho, \sigma}(f) D_{\rho, \sigma}
$$

Proof. Let $\delta$ denote a finite subset of $\mathcal{D}$, and let $\Delta$ denote the set of all such $\delta$. Put

$$
g_{\delta}=\sum_{D \in \delta} s^{D} \sum_{\tau}^{S^{D}} D_{\tau, \tau}
$$

Then $g_{\delta}$ is an almost periodic function [1, Theorem 6]. By [1, Theorem 22], for each $\delta \in \Delta$ we may find a sequence $\chi_{\delta, n}$ of special weight functions such that $\left|\chi_{\delta, n} \times g_{\delta}-g_{\delta}\right|<1 / n$. By [4, Theorem 21], $g_{\delta} \times D_{\rho, \sigma}=D_{\rho, \sigma}$ for all $D \in \delta$. Therefore

$$
\left|\chi_{\delta, n} \times D_{\rho, \sigma}-D_{\rho, \sigma}\right|<1 / n
$$

for all $D \in \delta$,

because, by [4, Theorems 11 and 7(5)], $\left|\chi_{\delta, n} \times g_{\delta} \times D_{\rho, \sigma}-g_{\delta} \times D_{\rho, \sigma}\right|$ $=\left|\left(\chi_{\delta, n} \times g_{\delta}-g_{\delta}\right) \times D_{\rho, \sigma}\right| \leqq\left|\chi_{\delta, n} \times g_{\delta}-g_{\delta}\right|<1 / n$.

If we denote by $\Gamma$ the set of all $\gamma=(\delta, n)$ with $\delta \in \Delta$ and $n$ a natural number, we obtain an index set $\Gamma$ which we may turn into a directed set by the following partial ordering: Put $\gamma_{1}=\left(\delta_{1}, n_{1}\right) \geqq \gamma_{2}=\left(\delta_{2}, n_{2}\right)$ if and only if $\delta_{1} \supseteq \delta_{2}$ and $n_{1} \geqq n_{2}$. Then from (4),

$$
\lim _{\gamma \in \Gamma} \chi_{\gamma} \times D_{\rho, \sigma}=D_{\rho, o} \quad \text { for all } D \in \mathcal{D}, 1 \leqq \rho, \sigma \leqq s^{D} \text {. }
$$

Put $\psi_{\gamma}=M_{y}\left\{\chi_{\gamma}\left(y x y^{-1}\right)\right\}$, so that $\psi_{\gamma}$ is a class function [1, Definition $10]$ and may be written [1, Theorem 29] as the finite sum

$$
\psi_{\gamma}=\sum_{D \in \mathcal{D}} s^{D} a_{D}^{\gamma} \sum_{\tau}^{\delta_{1}^{D}} D_{\tau \tau} \text { where } a_{D}^{\gamma}=\frac{1}{s^{D}} \sum_{\tau}^{\delta_{1}^{D}} C_{D, \tau, \tau}\left(\chi_{\gamma}\right),
$$

and only finitely many $a_{D}^{\gamma}$ are nonzero. Because of (5) we have that $\lim _{\gamma \in \Gamma} C_{D, \tau, \tau}\left(\chi_{\gamma}\right)=1$, and therefore $\lim _{\gamma \in \Gamma} a_{b}^{\gamma}=1$.

The function $\psi_{\gamma}^{\prime}$ defined by ${ }^{2}$

\footnotetext{
${ }^{2}[x]^{c}$ denotes the complex conjugate of $x$.
} 


$$
\psi_{\gamma}^{\prime}(x)=\left[\psi_{\gamma}\left(x^{-1}\right)\right]^{c}=\sum_{D \in \mathcal{D}} s^{D}\left[a_{D}^{\gamma}\right]^{c} \sum_{\tau}^{s^{D}} D_{\tau, \tau}(x)
$$

is also a special weight function. Put $\phi_{\gamma}=\psi_{\gamma} \times \psi_{\gamma}^{\prime}$. Then

$$
\phi_{\gamma}=\sum_{D \in \mathcal{D}} s^{D} r_{D}^{\gamma} \sum_{\tau}^{s_{1}^{D}} D_{\tau, \tau} \text { where } r_{D}^{\gamma}=\left|a_{D}^{\gamma}\right|^{2} \geqq 0
$$

and so $\lim _{\gamma \in \Gamma} r_{D}^{\gamma}=1$. Thus $\phi_{\gamma}$ is a class function [1, Theorem 29] and is also a special weight function [1, Theorem 18] and

$$
\begin{aligned}
0 & \leqq r_{D}=M_{x}\left\{D_{11}\left(x^{-1}\right) \phi_{\gamma}(x)\right\} \\
& \leqq M_{x}\left\{\left|D_{11}\left(x^{-1}\right)\right|\left|\phi_{\gamma}(x)\right|\right\} \leqq M_{x}\left\{\phi_{\gamma}(x)\right\}=1 .
\end{aligned}
$$

By $[1$, Theorem 24]

$$
\begin{aligned}
C_{D, \rho, \sigma}\left(\phi_{\gamma} \times f\right) & =\sum_{\tau}^{\delta_{1}^{D}} C_{D, \rho, \tau}\left(\phi_{\gamma}\right) C_{D, \tau, \sigma}(f) \\
& =\sum_{\tau}^{8}{ }_{1}^{D} r_{D}^{\gamma} \delta_{\rho, \tau} C_{D, \tau, \sigma}(f)=r_{D}^{\gamma} C_{D, \rho, \sigma}(f)
\end{aligned}
$$

so that $\phi_{\gamma} \times f=f_{\gamma}$ converges formally [1, Definition 9.] to the function $f$.

Thus we have to show that this formal convergence implies convergence. By $\left[1\right.$, Theorem 21] the set $\left\{f_{\gamma} \mid \gamma \in \Gamma\right\}$ is totally bounded and therefore has a compact closure. The directed set $\left\{f_{\gamma} \mid \gamma \in \Gamma\right\}$ therefore has a cluster point [6]. We show that it has only one cluster point. In fact suppose that $f^{\prime}$ and $f^{\prime \prime}$ are both cluster points. Then for a given $D, \rho, \sigma$, and $\epsilon>0$, because of the formal convergence, we may find $\gamma_{0} \in \Gamma$ such that for $\gamma^{\prime}, \gamma^{\prime \prime} \geqq \gamma_{0}$ we have that

$$
\left|C_{D, \rho, \sigma}\left(f_{\gamma^{\prime}}\right)-C_{D, \rho, \sigma}\left(f_{\gamma^{\prime \prime}}\right)\right|<\epsilon / 3 \text {. }
$$

But in particular we may choose $\gamma^{\prime}$ and $\gamma^{\prime \prime} \geqq \gamma_{0}$ so that $\left|f_{\gamma^{\prime}}-f^{\prime}\right|<\epsilon / 3$ and $\left|f_{\gamma^{\prime \prime}}-f^{\prime \prime}\right|<\epsilon / 3$, whence $\left|C_{D, \rho, \sigma}\left(f^{\prime}\right)-C_{D, \rho, \sigma}\left(f^{\prime \prime}\right)\right| \leqq \mid C_{D, \rho, \sigma}\left(f^{\prime}\right)$ $-C_{D, \rho, \sigma}\left(f_{\gamma^{\prime}}\right)|+| C_{D, \rho, \sigma}\left(f_{\gamma^{\prime}}\right)-C_{D ; \rho, \sigma}\left(f_{\gamma^{\prime \prime}}\right)|+| C_{D, \rho, \sigma}\left(f_{\gamma^{\prime \prime}}\right)-C_{D, \rho, \sigma}\left(f^{\prime \prime}\right) \mid$ $\leqq\left|f^{\prime}-f_{\gamma^{\prime}}\right|+\epsilon / 3+\left|f_{\gamma^{\prime \prime}}-f^{\prime \prime}\right|<\epsilon$. Thus $C_{D, \rho, \sigma} f^{\prime}=C_{D, \rho, \sigma} f^{\prime \prime}$. By the uniqueness theorem [1, Theorem 26], $f^{\prime}=f^{\prime \prime}=f$. Thus our conditionally compact directed set has exactly one cluster point $f$ and therefore converges to this cluster point. We may obtain (3) from (6). This concludes the proof of the theorem.

If we allow $\mathcal{D}$ to be the set of all irreducible, normal, unitary, and nonequivalent representations of $G$, then the functions $\phi_{\gamma}$ play the role of the Fejér kernel and the method of summation is applicable equally to all almost periodic functions on a group. 


\section{REFERENCES}

1. S. Bochner and J. von Neumann, Almost periodic functions in groups. II, Trans. Amer. Math. Soc. vol. 37 (1935) p. 21.

2. W. Maak, Abstrakte fastperiodische Funktionen, Abh. Math. Sem. Hansischen Univ. vol. 11 (1936) pp. 367-380.

3. - Summierung der Fourierreichen gleichartig fastperiodischer Funktionen auf Gruppen, Math. Zeit. vol. 52 (1950) pp. 770-778.

4. J. von Neumann, Almost periodic functions in a group. I, Trans. Amer. Math. Soc. vol. 36 (1934) pp. 445-492.

5. G. Birkhoff, Moore-Smith convergence in general topology, Ann. of Math. (2) vol. 38 (1937) pp. 39-57.

6. J. W. Tukey, Convergence and uniformity in topology, Annals of Mathematics Studies, no. 2, 1940.

BROWN UNIVERSITY 